UCRL-ID-135236

\title{
Surface Morphology Evolution in Silicon During Ion Beam Processing
}

\author{
T. Diaz de la Rubia \\ PJ. Bedrossian \\ MJ. Caturla \\ M.D. Johnson
}

August 1999

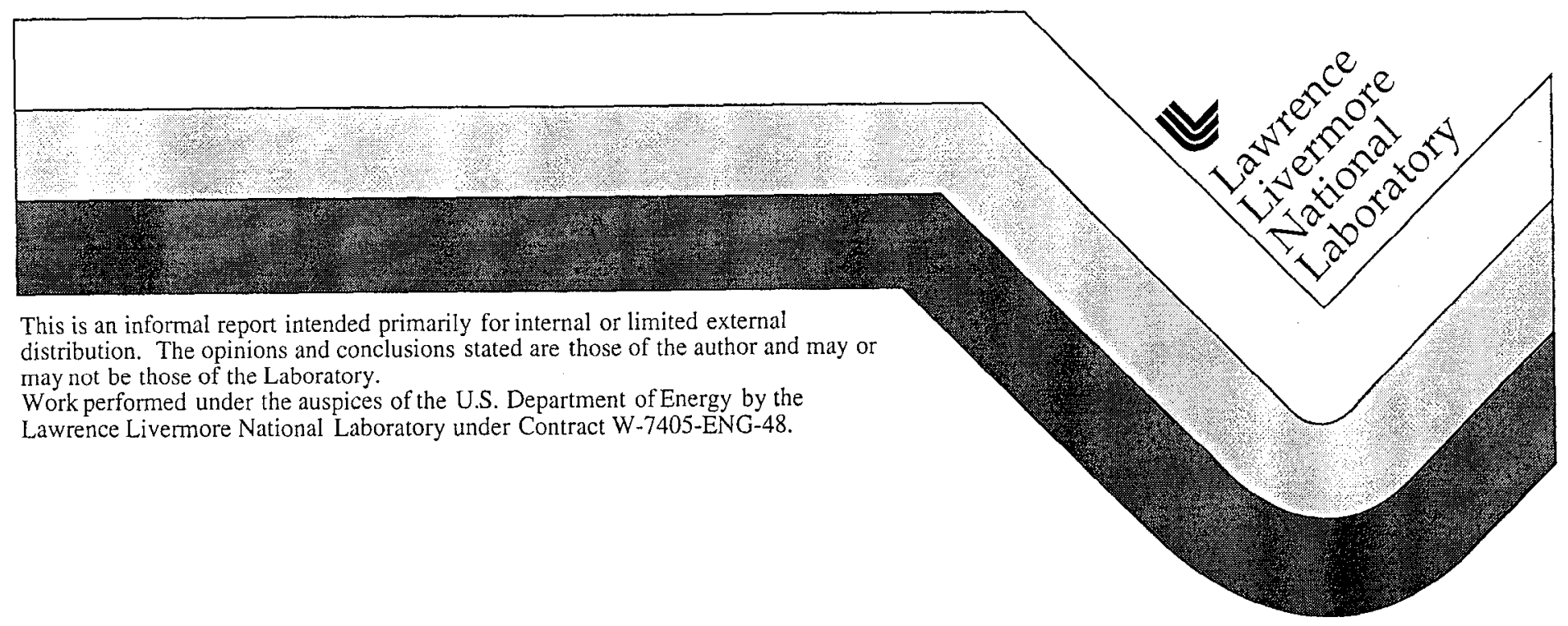




\section{DISCLAIMER}

This document was prepared as an account of work sponsored by an agency of the United States Government. Neither the United States Government nor the University of California nor any of their employees, makes any warranty, express or implied, or assumes any legal liability or responsibility for the accuracy, completeness, or usefulness of any information, apparatus, product, or process disclosed, or represents that its use would not infringe privately owned rights. Reference herein to any specific commercial product, process, or service by trade name, trademark, manufacturer, or otherwise, does not necessarily constitute or imply its endorsement, recommendation, or favoring by the United States Government or the University of California. The views and opinions of authors expressed herein do not necessarily state or reflect those of the United States Government or the University of California, and shall not be used for advertising or product endorsement purposes.

This report has been reproduced directly from the best available copy.

Available to DOE and DOE contractors from the Office of Scientific and Technical Information

P.O. Box 62, Oak Ridge, TN 37831

Prices available from (615) 576-8401, FTS 626-8401

Available to the public from the

National Technical Information Service

U.S. Department of Commerce

5285 Port Royal Rd.,

Springfield, VA 22161 


\title{
FINAL REPORT \\ 96-ERD-009
}

\author{
Surface Morphology Evolution in Silicon During Ion Beam Processing \\ PI: T. Diaz de la Rubia \\ Co-PI's: P.J. Bedrossian, M.J. Caturla and M.D. Johnson \\ Chemistry and Materials Science Directorate \\ Lawrence Livermore National Laboratory
}

The Semiconductor Industry Association (SIA) projccts that the semiconductor chips used in personal computers and scientific workstations will reach five times the speed and ten times the memory capacity of the current pentium-class processor by the year 2007. However, $1 \mathrm{GHz}$ on-chip clock speeds and 64 Gbits/Chip DRAM technology will not come easy and without a price. Such technologies will require scaling the minimum feature size of CMOS devices (the transistors in the silicon chip) down to below $100 \mathrm{~nm}$ from the current 180 to $250 \mathrm{~nm}$. This requirement has profound implications for device manufacturing. Existing processing techniques must increasingly be understood quantitatively and modeled with unprecedented precision. Indeed, revolutionary advances in the development of physics-based process simulation tools will be required to achieve the goals for cost efficient manufacturing, and to satisfy the needs of the defense industrial base. These advances will necessitate a fundamental improvement in our basic understanding of microstructure evolution during processing.

In order to cut development time and costs, the semiconductor industry makes extensive use of simple models of dopant implantation, and of phenomenological models of defect annealing and diffusion. However, the production of a single device often requires more than 200 processing steps, and the cumulative effects of the various steps are far too complex to be treated with these models. The lack of accurate process modeling simulators is proving to be a serious impediment to the development of next generation devices. New atomic-level models are required to describe the point defect distributions produced by the implantation process, and the defect and dopant diffusion resulting from rapid thermal annealing steps.

In this LDRD project, we investigated the migration kinetics of defects and dopants in silicon both experimentally and theoretically to provide a fundamental database for use in the development of predictive process simulators. The results were then used to develop kinetic Monte Carlo simulations that, when coupled to molecular dynamics studies, could be used to study and compare the long time and length scale behavior of ion implanted silicon to the predictions of experiments. The results of these kinetic Monte Carlo simulations were validated with experimental data and then used to predict boron activation fractions during annealing of ion implanted silicon under conditions similar to those encountered in the semiconductor manufacturing environment. The success of the work and promise of the approach are reflected in the number of publication and in the fact that following completion of the project we signed a funds-in CRADA with Intel

corporation and Applied Materials Corporation to continue the research. 
This report consists of a series of open literature publications that describe in chronological order the results obtained during the three years of this LDRD. The first publication, by M.J. Caturla; T. Diaz de la Rubia, L. Marques, and G.H. Gilmer, UCRLJC-122487, is entitled "Ion Beam Processing of Silicon at keV Energies: A Molecular Dynamics Study" and appeared in Physical Review B Vol. 54, 16683, 1996 (heretofore referred to as $\mathrm{P} 1$ ). This publication concerns the production of defects in the silicon lattice during the ion implantation process. This is a key issue since these defects represent the source term for all subsequent changes in the microstructure and the spatial distribution of the dopant concentration during the rapid thermal annealing process. The paper is a very detailed molecular dynamics study of the primary damage state in silicon following irradiation with boron and arsenic ions and presents a detail description of the amorphization process that ensues as a result of heavy ion irradiation of silicon.

The next paper is entitled "Intrinsic point defects in crystalline silicon: tight binding molecular dynamics studies of self-diffusion, interstitial-vacancy recombination, and formation volumes", UCRL-JC-124739, by M. Tang, L. Colombo, J. Zhu, and T. Diaz de la Rubia, and was published in the Physical Review B, vol. 55, 14279, 1997 (P2). This paper addresses the question of point defect properties in silicon. Key to modeling the long time scale evolution during high temperature annealing of the primary damage state following implantation (P1) is knowing the formation and migration enthalpies of the point defects produced. This paper addresses those questions with the use of tight binding electronic structure methods coupled with a molecular dynamics simulation algorithm. The results agree very well with the experimental database and demonstrate that self-diffusion in silicon at high temperatures is mediated by self-interstitial atoms, a point of debate in the scientific literature for over twenty years. Moreover, predictions are made as to the value of the equilibrium concentration of vacancies and selfinterstitials in silicon, a quantity that is not experimentally known. Most importantly, the paper demonstrates that in equilibrium vacancies diffuse faster than self-interstitials in silicon, a finding that at the time was counter to the commonly accepted believe that indeed the contrary was true.

Paper number three, "Damage evolution and surface defect segregation in low-energy ion-implanted silicon", UCRL-JC-125294, by P.J. Bedrossian, M.J. Caturla and T. Diaz de la Rubia, appeared in Applied Physics Letters, Vol. 70, 176, 1997 (P3). This paper applies a combination of experiments and simulations to validate the key finding of $\mathrm{P} 2$, namely that vacancies migrate faster than self interstitials in silicon at all temperatures. The paper is a combination of Scanning Tunneling Microscopy experiments and kinetic Monte Carlo simulations. Experimentally, we used atomically clean Si (111) surfaces and the Scanning Tunneling Microscope (STM) as monitors for the diffusion of implantationinduced vacancies and interstitials. The arrival of a vacancy (interstitial) at the surface causes the disappearance (reappearance) of a surface atom. We measured net arrival rates of vacancies and interstitials directly by using the STM to count the number of atoms populating the surface layer after various stages of annealing at different temperatures. We found that annealing at $350 \mathrm{C}$ results in a decrease in the atomic population of the adatom layer, while subsequent annealing at $500 \mathrm{C}$ restores the 
population of that layer. For the simulations, we used a combination of MD (P1) and kinctic Monte Carlo (KMC) simulations with kinetic data for point defects and clusters obtained in P2, to show that the experimental results can be explained by different rates of arrival to the surface of the vacancies and interstitials produced in the bulk during ion irradiation. The results of the simulations, which did not use any fitting parameters, were in excellent agreement with the experimental observations.

In a subsequent paper (P4), "Surface segregation and low-energy ion-induced defects in silicon", UCRL-JC-128754, J. Vacuum Science and Technology A, Vol. 16, 1043, 1998, by P. J. Bedrossian and T. Diaz de la Rubia, we extended the results of P3 by using $5 \mathrm{keV}$ helium ions as the irradiation agent in order to minimize the clustering of defects below the surface. The results were consistent with our previous simulations and served to further validate the findings of $\mathrm{P} 2$. In addition, the experiments showed that activation energies for bulk defect migration must be lower than for surface diffusion, a fact consistent with theoretical studies.

In the next paper (P5), we further validated our theory results by constructing a simulation aimed at explaining experimental data regarding the variability of the selfinterstitial diffusion coefficient with environmental conditions. The paper, UCRL-JC128774, was titled "A kinetic Monte Carlo study of the effective diffusivity of the silicon self interstitial in the presence of carbon and boron" by M.D. Johnson, M.J. Caturla and T. Diaz de la Rubia. In this paper, we showed that carbon and boron can act as traps for silicon self interstitials. In particular, we showed how varying concentrations of carbon in the lattice could affect not only the diffusivity but also the activation energy for migration of the defect. The simulation consisted of a two-stage trapping model and reproduced provided a very plausible explanation of the experimental database.

In the final paper (P6), we used the validated damage and defect property data to build a kinetic Monte Carlo simulation of boron implantation and high temperature annealing in silicon. The paper showed that our model agrees extremely well with experimental data for both the as implanted boron profile and the diffuse one obtained in experiments at Intel corporation following annealing a $700 \mathrm{C}, 800 \mathrm{C}$, and $900 \mathrm{C}$ for various times. Most importantly, the paper predicted that the fraction of substitutional and therefore electrically active during annealing goes through a maximum a few seconds after initiation of the anneal. This results, if proven correct, would potentially have significant consequences for silicon processing and device manufacturing since it would allow the process engineer to tune the length of the anneal to optimize the fraction of electrically active dopant during manufacturing. As a result of our studies, several groups around in the USA and Europe have started experiments aimed at validating our simulation predictions. This paper, UCRL-JC-129148, "The fraction of substitutional boron in silicon during ion implantation and thermal annealing" by M.J. Caturla, M.D. Johnson and T. Diaz de la Rubia was published in Applied Physics Letters, Vol. $72,2736,1998$.

In summary, over the duration of this LDRD we have constructed a model of ion implantation and rapid thermal annealing of silicon that is consistent with a widc array of 
experimental data. Moreover, our validated calculations and simulations have provided predictions that can be tested experimentally and that could potentially have significant impact in silicon technology development. As a result of this success, a funds-in CRADA with Intel and Applied Materials corporations is underway to further explore the application of our models. 